Jurnal Kimia Sains dan Aplikasi 24(5)(2021):170-176
ISSN: 1410-8917
Jurnal Kimia
Aplikasi
Journal KSN: 2597-9914

\title{
Natural Compounds Activities against SARS-CoV-2 Mpro through Bioinformatics Approaches for Development of Antivirus Candidates
}

\author{
Taufik Muhammad Fakih \\ Department of Pharmacy, Faculty of Mathematics and Natural Sciences, Universitas Islam Bandung, Bandung, Indonesia \\ Corresponding author: taufikmuhammadf@gmail.com \\ https://doi.org/10.14710/jksa.24.5.170-176
}

\section{Article Info}

Article history:

Received: $19^{\text {th }}$ May 2020

Revised: $28^{\text {th }}$ June 2021

Accepted: $26^{\text {th }}$ July 2021

Online: $31^{\text {st }}$ July 2021

Keywords:

COVID-19; SARS-CoV-2 Mpro; ACE-2; natural compounds; bioinformatics study

\begin{abstract}
Coronavirus infection (COVID-19) caused by SARS-CoV-2 appears as a pandemic that has spread to almost all countries in the world. Antiviral therapy using natural compounds is one alternative approach to overcome this infectious disease. The therapeutic mechanism is proven effective against the main protease (Mpro) of SARS-CoV-2. This research aims to perform bioinformatics studies, including ligand-docking simulations and protein-protein docking simulations, to identify, evaluate, and explore five compounds' activity on SARS-CoV-2 Mpro and their effects against Angiotensin-Converting Enzyme 2 (ACE-2). Proteinligand docking simulations show kaempferol, flavonol, and their glycosides (Afzelin and Juglanin) and other flavonoids (Quercetin, Naringenin, and Genistein) have a high affinity towards SARS-CoV-2 Mpro. These results were then confirmed using protein-protein docking simulations to observe the ability of five compounds to prevent the attachment of ACE-2 to the active site. Based on the results of the bioinformatics studies, Quercetin has the best affinity, with a binding free energy value of $-33.18 \mathrm{~kJ} / \mathrm{mol}$. The five compounds are predicted to be able to interact strongly with SARS-CoV-2. The results in this research are useful for further studies in the development of novel anti-infective drugs for COVID-19 that target SARS-CoV-2 Mpro.
\end{abstract}

\section{Introduction}

The 2019 coronavirus infection (COVID-19) was first discovered in Wuhan City, Hubei Province, China, and has now become a pandemic due to its rapid spread to 25 countries worldwide [1]. For this incident, the World Health Organization (WHO) announced a global health emergency on 30 January 2020 [2, 3]. In its first emergency meeting, WHO estimated the COVID-19 mortality rate to be around 4\% [4]. Collaborative efforts by researchers worldwide are underway to understand the characteristics of the virus that causes this disease, namely SARS-CoV-2 (originally named 2019-nCoV), and develop effective drug candidates to control and prevent it [5].
The SARS-CoV-2 genome consists of $\sim 30,000$ nucleotides and functions to encode two overlapping polyproteins, namely pp1a and pp1ab. These polyproteins are needed for virus replication and transcription [6]. Functional polypeptides are released from polyproteins through proteolytic processes involving the main protease (Mpro) [7]. Preliminary studies also show that SARS-CoV-2 has structural similarities to SARS-CoV based on complete phylogenetic analysis of the genome $[8,9]$. Besides, both interact directly with AngiotensinConverting Enzyme 2 (ACE-2) to enter the target cell [10].

ACE- 2 can mediate the entry of SARS-CoV-2 into cells so that it acts as a functional receptor for coronavirus. SARS-CoV-2 involves ACE-2 with an affinity 
comparable to SARS-CoV [11]. The tight bonding in ACE2 can explain part of the efficient transmission of SARSCoV-2 in humans, as happened in SARS-CoV [12]. Therefore, the inhibition of SARS-CoV-2 attachment to ACE-2 is a pathway in developing inhibitors for COVID-19 infectious diseases.

Several herbal antiviral agents have been developed to disrupt the viral life cycle [13]. At the beginning of the first appearance of SARS in 2002-2003, around $50 \%$ of patients in mainland China were successfully treated using herbal medicines [13]. Kaempferol flavonols and their glycosides (Afzelin and Juglanin), as well as other flavonoids (Quercetin, Naringenin, and Genistein), have been reported to have inhibitory activity against the main protease (Mpro) SARS-CoV or viral replication [14]. Interestingly, all of these compounds are contained in natural Indonesian herbs, such as Afzelin (from Annona purpurea, Piper umbellatum, Zingiber zerumbet, Nymphaea odorata, and Ginkgo biloba) [15], Juglanin (from Polygonum aviculare) [16, 17], Quercetin (from Camellia sinensis, Moringa oleifera, Centella asiatica, Apium graveolens, and Coriandrum sativum) [18], Naringenin (Citrus species, Ficus carica) [19], Genistein (Flemingia vestita, Rutaceae family, Fortunella obovata, Erythrina variegata, Millettia reticulata, Tetracera scandens, Genista sessilifolia, and Amaryllidaceae species) [20].
Nowadays, the need to design effective antiviral candidates against SARS-CoV-2 is increasing. This research aims to observe the molecular interactions between several natural compounds in the main protease (Mpro) of SARS-CoV-2 and their effects in inhibiting the binding of ACE-2. Bioinformatics approaches through ligand-protein, and protein-protein docking simulations can be used to identify, evaluate, and characterize potential components of SARS-COV-2 [21]. Specifically, SARS-CoV-2 Mpro is considered a target because it is a major part of forming the characteristics of the coronavirus. Thus, through this research, it is expected to obtain the structure of reference compounds to treat COVID-19 infections.

\section{Methodology}

\subsection{Macromolecule Preparation}

Macromolecules used in this research were the main proteases (Mpro) of SARS-CoV-2, and AngiotensinConverting Enzyme (ACE-2) obtained from Protein Data Bank (http://www.rcsb.org/pdb) with PDB ID 6LU7 [22] and 2AJF [23], respectively. The preparation of these two macromolecules was carried out by removing water molecules and native ligands, adding polar hydrogen atoms, and calculating the Kollman charge using AutoDock 4.2 with MGLTools 1.5.6 [24, 25].

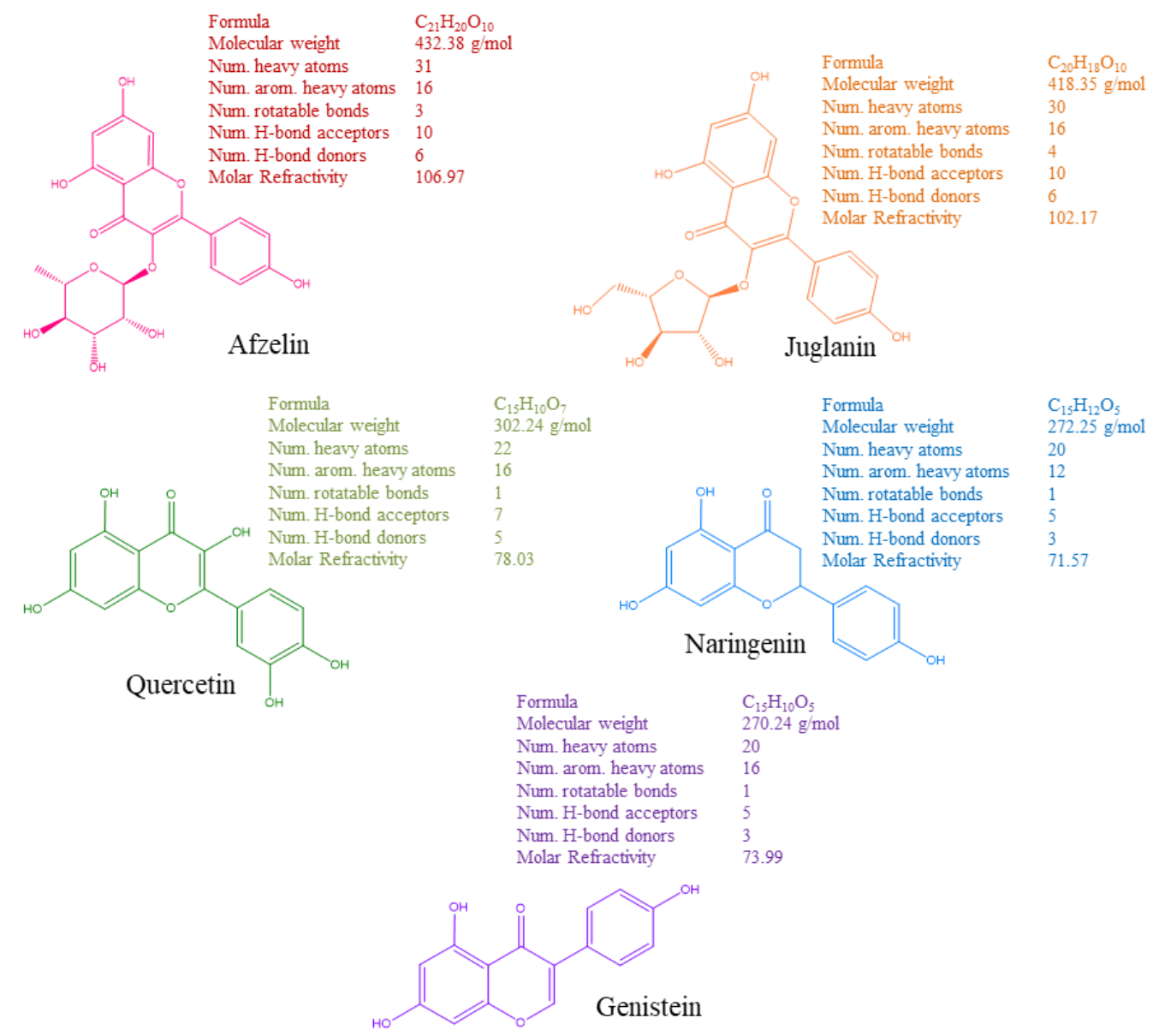

Figure 1. Two-dimensional structure and physicochemical properties of ligands 


\subsection{Ligand Preparation}

The ligands used in this research were Kaempferol flavonol and their glycosides (Afzelin and Juglanin), as well as other flavonoids (Quercetin, Naringenin, and Genistein), obtained from PubChem (https://pubchem.ncbi.nlm.nih.gov/) (Figure 1). The ligand structure was optimized using Quantum ESPRESSO 6.6 with Density Functional Theory (DFT) method based on the $3-21 \mathrm{G}$ basis set $[26,27]$. Finally, all ligands were prepared by adding a hydrogen atom and calculating the Gasteiger charge using AutoDock 4.2 with MGLTools 1.5.6 [24, 25]. Structural preparation aims to optimize compounds for use as input in ligand-protein docking simulations.

\subsection{Identification of Ligand-Binding Sites in SARS- CoV-2 Mpro}

Macromolecules of SARS-CoV-2 Mpro that have been prepared are then identified, evaluated, and explored binding sites that are most responsible for biological activity using BIOVIA Discovery Studio 2020 [28]. All amino acids that occur around the native ligand within a spherical radius distance set are prepared for the location of protein-ligand binding in ligand-protein docking simulations.

\subsection{Ligand-Protein Docking Simulations}

The ligand-protein docking simulations were performed using AutoDock 4.2 with MGLTools 1.5.6 [24, 25]. All ligand molecules for this simulation were added hydrogen atoms and partial charges data from the Density Functional Theory (DFT) calculations. Simulations were created by a grid box $64 \times 60 \times 60$ points with $0.375 \AA$ spacing to cover the binding cavity of the target [29]. The Lamarckian Genetic Algorithm with 100 conformations was chosen for each simulation, and other docking parameters were set as default. Observation of the docking simulation results was performed using Discovery Studio 2020 [28].

\subsection{Protein-Protein Docking Simulations}

Protein-protein docking simulations were performed using PatchDock for five complexes from the results of ligand-protein docking simulations in SARSCoV-2 Mpro [30]. The simulations were accomplished against active sites of the Angiotensin-Converting Enzyme (ACE-2) macromolecules, including Gln24, Lys31, His34, Glu37, Asp38, Tyr41, Gln42, Met82, Tyr83, Glu329, Asn330, Lys353, and Gly354. The default clustering RMSD $4.0 \AA$ was used, and the complex type was chosen to be a protein-protein. The Connolly dot surface representation of the molecules into different components such as convex, concave, and flat patches were generated through the PatchDock algorithm. PatchDock was optimized, refined, reshuffled, and rescored the side chain interface of the top 10 candidate solutions. It also amends the orientation of the relative molecules by confining the flexibility to the side-chains of the interacting surface and allow the movements of a small rigid body. The conformation of the systems was verified by visualization analysis with Discovery Studio 2020 [28].

\section{Results and Discussions}

\subsection{Ligand-Protein Docking Simulations}

Kaempferol flavonol and their glycosides (Afzelin and Juglanin) and other flavonoids (Quercetin, Naringenin, and Genistein) were docked into SARS-CoV2 Mpro as a macromolecular target. Moreover, molecular docking of natural ligands was also performed as a comparison against the five tested ligands. All complexes from the results from protein-ligand docking were selected for further studies using protein-protein docking methods against ACE-2. Five ligands have a larger negative binding free energy than natural ligands to the active site of SARS-CoV-2 Mpro (Table 1). This phenomenon shows a promising sign that these ligands have an excellent affinity to the target macromolecules. Quercetin has the best binding at the active site of SARS$\mathrm{CoV}-2$, with a binding free energy value of $-33.18 \mathrm{~kJ} / \mathrm{mol}$ better than other ligands.

Table 1. Binding Free Energy of Ligands to SARS-CoV-2 Mpro

\begin{tabular}{cc}
\hline Ligand & Binding Free Energy $(\mathrm{kJ} / \mathrm{mol})$ \\
\hline Native ligand & -18.58 \\
Afzelin & -27.49 \\
Juglanin & -32.05 \\
Quercetin & -33.18 \\
Naringenin & -30.46 \\
Genistein & -29.33 \\
\hline
\end{tabular}

All the ligands have related interactions against SARS-CoV-2 Mpro (indicated by the binding energy value, which is almost similar) (Figure 2). In general, the interaction of each ligand with SARS-CoV-2 Mpro was dominated by ten hydrogen bonds (with Phe140, Gly143, His163, His164, Met165, Glu166, His172, Gln189, and Thr190) and seven hydrophobic interactions (with His41, Met49, Leu141, Asn142, Met165, Pro168, and Ala191). Interestingly, Quercetin, as the ligand with the best binding free energy, has more molecular interactions than natural ligands. Quercetin can form twelve interactions, including seven hydrogen bonds (with Gln192, Thr190, Thr190, Glu166, His164, Tyr54, and Asp187) and five hydrophobic interactions (with His41, Met165, Met165, Met165, and Pro168). Meanwhile, natural ligands could only form ten interactions consisting of seven hydrogen bonds (with Asn142, Phe140, Glu166, Gln189, Thr190, Asn142, and Leu141) and three hydrophobic interactions (with Met165, Pro168, and Ala191). 


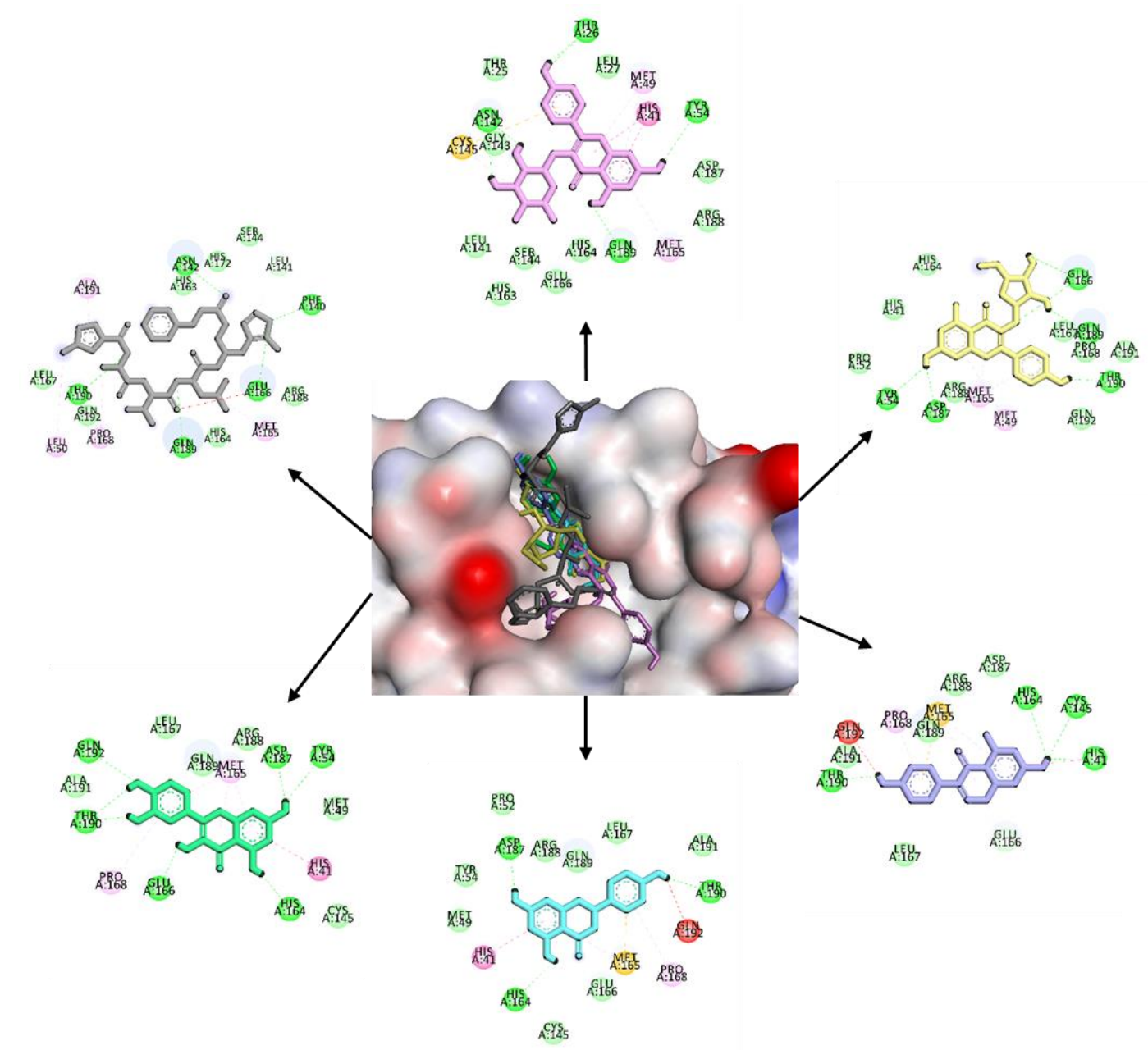

Figure 2. Overlay the docking pose of Afzelin (pink), Juglanin (yellow), Quercetin (green), Naringenin (blue), Genistein (purple), and natural ligand (gray) in the binding site of SARS-CoV-2 Mpro

The molecular interactions that form between molecular ligands and the active site of the macromolecular target consisted of many hydrogen bonds. These ligands act as donor hydrogen bonds and protein amino acid residues as hydrogen bond acceptors. Most hydrogen bonds between protein-ligands were quite strong, with an average bond length below $3 \AA$. Due to the presence of hydrogen bonds, the interaction between five ligands and SARS-CoV-2 Mpro was also dominated by hydrophobic interactions [31]. It can be predicted that hydrogen bonds and hydrophobic interactions that contribute against macromolecules protein play an important role in stabilizing the complex. The best conformation of ligands to the SARS-CoV-2 Mpro receptor was chosen for performing the proteinprotein docking simulations.

\subsection{Protein-Protein Docking Simulations}

After ligand-docking simulations, further identifications were conducted using protein-protein docking methods. The atomic contact energy evaluated the ACE-2 (ACE) score's binding affinity into the PatchDock [30]. The purpose of such docking simulations was to examine the bonding effect of each ligand with the target protein in preventing the attachment of ACE- 2 to the active site of SARS-CoV-2 Mpro. The preparation of
ACE-2 macromolecules was demonstrated by removing water molecules and native ligands, adding polar hydrogen atoms, and calculating the Kollman charge using AutoDock 4.2 with MGLTools 1.5.6 [24, 25]. A strong ligand bond to the target macromolecule is predicted to tend to inhibit the entry of coronavirus into cells due to the inability of SARS-CoV-2 Mpro to reach ACE-2 to forward the signal [32]. Therefore, the exploration of amino acid residues responsible for arresting the formation of molecular interactions between the binding sites of SARS-CoV-2 Mpro and ACE-2 is also needed.

Table 2. Atomic Contact Energy of Each Ligand-Protein Complex to ACE-2 in Protein-Protein Docking

\begin{tabular}{cc}
\hline Ligand-Protein Complex & $\begin{array}{c}\text { Atomic Contact Energy } \\
(\mathrm{kJ} / \mathrm{mol})\end{array}$ \\
\hline SARS-CoV-2 Mpro & -1172.98 \\
Afzelin + SARS-CoV-2 Mpro & 1321.39 \\
Juglanin + SARS-CoV-2 Mpro & 1532.14 \\
Quercetin + SARS-CoV-2 & 1684.48 \\
Mpro & 1676.91 \\
Naringenin + SARS-CoV-2 & \\
Mpro & 1654.90 \\
Genistein + SARS-CoV-2 & \\
Mpro & 1321.39 \\
Afzelin + SARS-CoV-2 Mpro & \\
\hline
\end{tabular}


Data from protein-protein docking simulation shows that five ligand-protein complexes have positive ACE scores (Table 2). This occurrence can be caused by unfavorable interactions between each ligand-protein complex against ACE-2 [33]. Interestingly, Quercetin effectively inhibited the attachment of ACE- 2 to the binding area of SARS-CoV-2 Mpro because it has the largest positive ACE, with a score of $1684.48 \mathrm{~kJ} / \mathrm{mol}$. Most inhibitors work by binding firmly to the active sites of the target receptor and competing with its native ligand, as well as stabilizing the structure of the receptor macromolecules and preventing the conformational changes needed to progress the signal further.

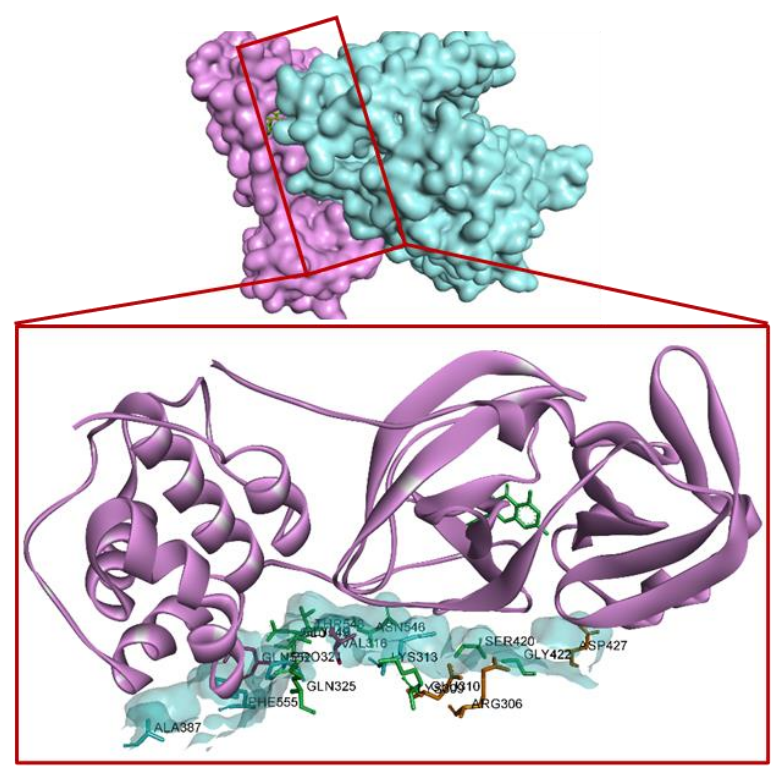

- Unfavorable interaction, Hydrogen bond,

- Hydrophobic interaction, Electrostatic interaction

Figure 3. Protein-Protein Docking Pose of Quercetin Complexes (pink) against ACE-2 (blue)

Subsequent analysis was performed on the conformation of the Quercetin + SARS-CoV-2 Mpro complex with ACE-2 (Figure 3). Ten unfavorable bonds were formed, namely the amino acid residues Lys313, Val316, Val316, Ala387, Gln552, Gln552, Gln552, Gln552, Phe555, and Pro321. Besides, other interactions that contribute include thirteen hydrogen bonds (with Arg306, Asp427, Glu310, Lys309, Thr548, Gln325, Thr548, Glu549, Asn546, Gly319, Gly319, Ser420, and Gly422), six hydrophobic interactions (with Lys313, Pro321, Ala387, Lys313, Pro321, and Phe555), and nine electrostatic interactions (with Arg306, Asp427, Asp427, Glu310, Glu310, Asp427, Arg306, Lys313, and Phe555). Eventually, it can be predicted that Quercetin is a potential candidate for SARS-CoV-2 Mpro inhibitor and can prevent attachment to ACE-2, which acts as an entry point into human cells.

\section{Conclusion}

Several compounds of kaempferol, flavonol, and glycoside derivatives (Afzelin and Juglanin) and other flavonoids (Quercetin, Naringenin, and Genistein) can bind stably against SARS-CoV-2 Mpro. However, Quercetin has the strongest interaction with the target macromolecular active site, with the binding free energy value of $-33.18 \mathrm{~kJ} / \mathrm{mol}$. The five ligands are also able to inhibit the formation of interactions with ACE-2. Thus, the study results indicate that the compound can be further developed as a SARS-CoV-2 Mpro inhibitor in COVID-19 infection therapy.

\section{Acknowledgment}

The authors thank the LPPM (Institute for Research and Community Service), Universitas Islam Bandung, for the research financially supported by the Special Research Grant Program 2020, No.039/B.04/LPPM/IV/2020.

\section{References}

[1] Chen Wang, Peter W. Horby, Frederick G. Hayden, George F. Gao, A novel coronavirus outbreak of global health concern, The Lancet, 395, 10223, (2020), 470-473 https://doi.org/10.1016/S0140-6736(20)30185-9

[2] Chaolin Huang, Yeming Wang, Xingwang Li, Lili Ren, Jianping Zhao, Yi Hu, Li Zhang, Guohui Fan, Jiuyang Xu, Xiaoying Gu, Zhenshun Cheng, Ting Yu, Jiaan Xia, Yuan Wei, Wenjuan Wu, Xuelei Xie, Wen Yin, Hui Li, Min Liu, Yan Xiao, Hong Gao, Li Guo, Jungang Xie, Guangfa Wang, Rongmeng Jiang, Zhancheng Gao, Qi Jin, Jianwei Wang, Bin Cao, Clinical features of patients infected with 2019 novel coronavirus in Wuhan, China, The Lancet, 395, 10223, (2020), 497-506 https://doi.org/10.1016/S0140-6736(20)30183-5

[3] David L. Heymann, Data sharing and outbreaks: best practice exemplified, The Lancet, 395, 10223, (2020), 469-470 https://doi.org/10.1016/S0140-6736(20)30184-7

[4] Catrin Sohrabi, Zaid Alsafi, Niamh O'Neill, Mehdi Khan, Ahmed Kerwan, Ahmed Al-Jabir, Christos Iosifidis, Riaz Agha, World Health Organization declares global emergency: A review of the 2019 novel coronavirus (COVID-19), International Journal of Surgery, 76, (2020), 71-76

https://doi.org/10.1016/j.ijsu.2020.02.034

[5] Peng Zhou, Xing-Lou Yang, Xian-Guang Wang, Ben Hu, Lei Zhang, Wei Zhang, Hao-Rui Si, Yan Zhu, Bei Li, Chao-Lin Huang, Hui-Dong Chen, Jing Chen, Yun Luo, Hua Guo, Ren-Di Jiang, Mei-Qin Liu, Ying Chen, Xu-Rui Shen, Xi Wang, Xiao-Shuang Zheng, Kai Zhao, Quan-Jiao Chen, Fei Deng, Lin-Lin Liu, Bing Yan, Fa-Xian Zhan, Yan-Yi Wang, Geng-Fu Xiao, Zheng-Li Shi, A pneumonia outbreak associated with a new coronavirus of probable bat origin, Nature, 579, 7798, (2020), 270-273 https://doi.org/10.1038/s41586-020-2012-7

[6] Roujian Lu, Xiang Zhao, Juan Li, Peihua Niu, Bo Yang, Honglong Wu, Wenling Wang, Hao Song, Baoying Huang, Na Zhu, Yuhai Bi, Xuejun Ma, Faxian Zhan, Liang Wang, Tao Hu, Hong Zhou, Zhenhong Hu, Weimin Zhou, Li Zhao, Jing Chen, Yao Meng, Ji Wang, Yang Lin, Jianying Yuan, Zhihao Xie, Jinmin Ma, William J. Liu, Dayan Wang, Wenbo Xu, Edward 
C. Holmes, George F. Gao, Guizhen Wu, Weijun Chen, Weifeng Shi, Wenjie Tan, Genomic characterisation and epidemiology of 2019 novel coronavirus: implications for virus origins and receptor binding, The Lancet, 395, 10224, (2020), 565-574 https://doi.org/10.1016/S0140-6736(20)30251-8

[7] Xing-Yi Ge, Jia-Lu Li, Xing-Lou Yang, Aleksei A. Chmura, Guangjian Zhu, Jonathan H. Epstein, Jonna K. Mazet, Ben Hu, Wei Zhang, Cheng Peng, Yu-Ji Zhang, Chu-Ming Luo, Bing Tan, Ning Wang, Yan Zhu, Gary Crameri, Shu-Yi Zhang, Lin-Fa Wang, Peter Daszak, Zheng-Li Shi, Isolation and characterization of a bat SARS-like coronavirus that uses the ACE2 receptor, Nature, 503, 7477, (2013), 535-538 https://doi.org/10.1038/nature12711

[8] Michael Letko, Andrea Marzi, Vincent Munster, Functional assessment of cell entry and receptor usage for SARS-CoV-2 and other lineage B betacoronaviruses, Nature Microbiology, 5, 4, (2020), 562-569 https://doi.org/10.1038/s41564-020-0688-y

[9] Yu Chen, Hui Cai, Ji Pan, an, Nian Xiang, Po Tien, Tero Ahola, Deyin Guo, Functional screen reveals SARS coronavirus nonstructural protein nsp14 as a novel cap N7 methyltransferase, Proceedings of the National Academy of Sciences, 106, 9, (2009), 3484 https://doi.org/10.1073/pnas.0808790106

[10] Alexandra C. Walls, Young-Jun Park, M. Alejandra Tortorici, Abigail Wall, Andrew T. McGuire, David Veesler, Structure, Function, and Antigenicity of the SARS-CoV-2 Spike Glycoprotein, Cell, 181, 2, (2020), 281-292.e286

https://doi.org/10.1016/j.cell.2020.02.058

[11] Alexandra C. Walls, Xiaoli Xiong, Young-Jun Park, M. Alejandra Tortorici, Joost Snijder, Joel Quispe, Elisabetta Cameroni, Robin Gopal, Mian Dai, Antonio Lanzavecchia, Maria Zambon, Félix A. Rey, Davide Corti, David Veesler, Unexpected Receptor Functional Mimicry Elucidates Activation of Coronavirus Fusion, Cell, 176, 5, (2019), 10261039.e1015 https://doi.org/10.1016/j.cell.2018.12.028

[12] Jung-Eun Park, Kun Li, Arlene Barlan, Anthony R. Fehr, Stanley Perlman, Paul B. McCray, Tom Gallagher, Proteolytic processing of Middle East respiratory syndrome coronavirus spikes expands virus tropism, Proceedings of the National Academy of Sciences, 113, 43, (2016), 12262

https://doi.org/10.1073/pnas.1608147113

[13] Kai Wang, Shiqi Xie, Bing Sun, Viral proteins function as ion channels, Biochimica et Biophysica Acta (BBA) - Biomembranes, 1808, 2, (2011), 510-515 https://doi.org/10.1016/j.bbamem.2010.05.006

[14] Silvia Schwarz, Kai Wang, Wenjing Yu, Bing Sun, Wolfgang Schwarz, Emodin inhibits current through SARS-associated coronavirus 3a protein, Antiviral Research, 90, 1, (2011), 64-69 https://doi.org/10.1016/j.antiviral.2011.02.008

[15] Eva Rachmi, Basuki Bambang Purnomo, Agustina Tri Endharti, Loeki Enggar Fitri, Identification of afzelin potential targets in inhibiting triplenegative breast cancer cell migration using reverse docking, Porto Biomedical Journal, 5, 6, (2020), e095 https://doi.org/10.1097/j.pbj.0000000000000095
[16] Liang Chen, Ya-Qiong Xiong, Jing Xu, Ji-Peng Wang, Zi-Li Meng, Yong-Qing Hong, Juglanin inhibits lung cancer by regulation of apoptosis, ROS and autophagy induction, Oncotarget, 8, 55, (2017), 93878-93898

https://doi.org/10.18632/oncotarget.21317

[17] Ze-Wu Dong, Yu-Fang Yuan, Juglanin suppresses fibrosis and inflammation response caused by LPS in acute lung injury, International Journal of Molecular Medicine, 41, 6, (2018), 3353-3365

https://doi.org/10.3892/ijmm.2018.3554

[18] A. V. Anand David, R. Arulmoli, S. Parasuraman, Overviews of Biological Importance of Quercetin: A Bioactive Flavonoid, Pharmacognosy Reviews, 10, 20, (2016), 84-89

https://doi.org/10.4103/0973-7847.194044

[19] Bahare Salehi, Patrick Valere Tsouh Fokou, Mehdi Sharifi-Rad, Paolo Zucca, Raffaele Pezzani, Natália Martins, Javad Sharifi-Rad, The Therapeutic Potential of Naringenin: A Review of Clinical Trials, Pharmaceuticals, 12, 1, (2019), 11 https://doi.org/10.3390/ph12010011

[20] V. Tandon, B. Das, Genistein: is the multifarious botanical a natural anthelmintic too?, Journal of Parasitic Diseases, 42, 2, (2018), 151-161 https://doi.org/10.1007/s12639-018-0984-0

[21] Swatantra Kumar, Vimal K. Maurya, Anil K. Prasad, Madan L. B. Bhatt, Shailendra K. Saxena, Structural, glycosylation and antigenic variation between 2019 novel coronavirus (2019-nCoV) and SARS coronavirus (SARS-CoV), VirusDisease, 31, 1, (2020), 13-21 https://doi.org/10.1007/s13337-020-00571-5

[22] Zhenming Jin, Xiaoyu Du, Yechun Xu, Yongqiang Deng, Meiqin Liu, Yao Zhao, Bing Zhang, Xiaofeng Li, Leike Zhang, Chao Peng, Yinkai Duan, Jing Yu, Lin Wang, Kailin Yang, Fengjiang Liu, Rendi Jiang, Xinglou Yang, Tian You, Xiaoce Liu, Xiuna Yang, Fang Bai, Hong Liu, Xiang Liu, Luke W. Guddat, Wenqing Xu, Gengfu Xiao, Chengfeng Qin, Zhengli Shi, Hualiang Jiang, Zihe Rao, Haitao Yang, Structure of Mpro from SARS-CoV-2 and discovery of its inhibitors, Nature, 582, 7811, (2020), 289-293 https://doi.org/10.1038/s41586-020-2223-y

[23] Fang Li, Wenhui Li, Michael Farzan, Stephen C. Harrison, Structure of SARS Coronavirus Spike Receptor-Binding Domain Complexed with Receptor, Science, 309, 5742, (2005), 1864 https://doi.org/10.1126/science.1116480

[24] David S. Goodsell, Garrett M. Morris, Arthur J. Olson, Automated docking of flexible ligands: Applications of autodock, Journal of Molecular Recognition, 9, 1, (1996), 1-5 https://doi.org/10.1002/(SICI)10991352(199601)9:1<1::AID-JMR241>3.0.CO;2-6

[25] Garrett M. Morris, David S. Goodsell, Robert S. Halliday, Ruth Huey, William E. Hart, Richard K. Belew, Arthur J. Olson, Automated docking using a Lamarckian genetic algorithm and an empirical binding free energy function, Journal of Computational Chemistry, 19, 14, (1998), 1639-1662 https://doi.org/10.1002/(SICI)1096987X(19981115)19:14<1639::AID-JCC10>3.0.CO;2-B 
[26] Paolo Giannozzi, Stefano Baroni, Nicola Bonini, Matteo Calandra, Roberto Car, Carlo Cavazzoni, Davide Ceresoli, Guido L. Chiarotti, Matteo Cococcioni, Ismaila Dabo, Andrea Dal Corso, Stefano de Gironcoli, Stefano Fabris, Guido Fratesi, Ralph Gebauer, Uwe Gerstmann, Christos Gougoussis, Anton Kokalj, Michele Lazzeri, Layla Martin-Samos, Nicola Marzari, Francesco Mauri, Riccardo Mazzarello, Stefano Paolini, Alfredo Pasquarello, Lorenzo Paulatto, Carlo Sbraccia, Sandro Scandolo, Gabriele Sclauzero, Ari P. Seitsonen, Alexander Smogunov, Paolo Umari, Renata M. Wentzcovitch, QUANTUM ESPRESSO: a modular and open-source software project for quantum simulations of materials, Journal of Physics: Condensed Matter, 21, 39, (2009), 395502

http://dx.doi.org/10.1088/0953-8984/21/39/395502

[27] Paolo Giannozzi, Oscar Baseggio, Pietro Bonfà, Davide Brunato, Roberto Car, Ivan Carnimeo, Carlo Cavazzoni, Stefano de Gironcoli, Pietro Delugas, Fabrizio Ferrari Ruffino, Andrea Ferretti, Nicola Marzari, Iurii Timrov, Andrea Urru, Stefano Baroni, Quantum ESPRESSO toward the exascale, The Journal of Chemical Physics, 152, 15, (2020), 154105 https://doi.org/10.1063/5.0005082

[28] Devjani I. Banerjee, Tejas P. Gohil, Interaction of antimicrobial peptide with mycolyl transferase in Mycobacterium tuberculosis, International Journal of Mycobacteriology, 5, 1, (2016), 83-88 https://doi.org/10.1016/j.ijmyco.2015.07.002

[29] Meshari Alazmi, Olaa Motwalli, In silico virtual screening, characterization, docking and molecular dynamics studies of crucial SARS-CoV-2 proteins, Journal of Biomolecular Structure and Dynamics, (2020), 1-11

https://doi.org/10.1080/07391102.2020.1803965

[30] D. Sathya Prabhu, V. Devi Rajeswari, In Silico Docking Analysis of Bioactive Compounds from Chinese Medicine Jinqi Jiangtang Tablet (JOJTT) using Patch Dock, Journal of Chemical and Pharmaceutical Research, 8, 5, (2016), 15-21

[31] Dwi Syah Fitra Ramadhan, Taufik Muhammad Fakih, Arfan Arfan, Activity Prediction of Bioactive Compounds Contained in Etlingera elatior Against the SARS-CoV-2 Main Protease: An In Silico Approach, Borneo Journal of Pharmacy, 3, 4, (2020), 235-242 https://doi.org/10.33084/bjop.v3i4.1634

[32] F. Fitriyani, Taufik Muhammad Fakih, Daryono Hadi Tjahjono, In Silico Studies of Green Tea CatechinsAgainst HER-2 Receptor in Breast Cancer, Current Trends in Biotechnology \& Pharmacy, 14, 5, (2020), 194-199 https://doi.org/10.5530/ctbp.2020.4s.23

[33] Vijayakumar Veeraragavan, Radhakrishnan Narayanaswamy, Rameshkumar Chidambaram, Predicting the Biodegradability Nature of Imidazole and Its Derivatives by Modulating Two Histidine Degradation Enzymes (Urocanase and Formiminoglutamase) Activities, Asian Journal of Pharmaceutical and Clinical Research, 10, 11, (2017), 383-386 https://doi.org/10.22159/ajpcr.2017.v10i11.20999 